\title{
Continuidad pedagógica en Pandemia. Un estudio sobre la intensificación del trabajo docente en contextos de desigualdad
}

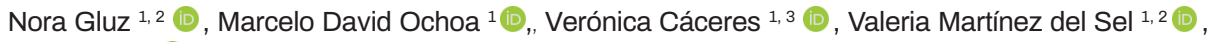 \\ Pablo Sisti ${ }^{1}$ (i) \\ ${ }^{1}$ Universidad Nacional de General Sarmiento (UNGS), ${ }^{2}$ Universidad de Buenos Aires (UB), ${ }^{3}$ Consejo Nacional \\ de Investigaciones Científicas y Técnicas, Argentina
}

Resumen. La emergencia sanitaria y la suspensión de las clases presenciales conforman un escenario complejo que en la provincia de Buenos Aires se agrava por sus profundas asimetrías históricas. En este contexto, se presenta un análisis de las condiciones de trabajo docente en la pandemia en la educación secundaria, desarrollando en primer lugar, una caracterización crítica sobre la dimensión socioeconómica y ambiental del territorio bonaerense. En segundo lugar, se analizan las políticas de continuidad pedagógica y la regulación del trabajo docente en una situación de excepcionalidad. Por último, y en vistas a que el sostenimiento de la continuidad pedagógica tuvo lugar en un contexto de clara dilución de las fronteras entre el trabajo docente y la vida doméstica en una profesión altamente feminizada; se señalan las principales tensiones entre las condiciones sociosanitarias, las regulaciones del trabajo docente y las condiciones de género que afectan las dinámicas que articulan el derecho a la educación y los derechos laborales. El análisis es producto de un estudio en curso que propone caracterizar las heterogeneidades territoriales y profundizar su incidencia sobre el trabajo docente a través de fuentes secundarias y de una encuesta y entrevistas en profundidad en cuatro localidades que expresan diferentes condiciones sociosanitarias.

Palabras clave: política educativa; trabajo docente; COVID-19; provincia de Buenos Aires.

Continuidade pedagógica na Pandemia. Um estudo sobre a intensificação do trabalho docente em contextos de desigualdade

Resumo. A emergência sanitária e a suspensão das aulas presenciais compõem um cenário complexo que na província de Buenos Aires é agravado por suas profundas assimetrias históricas. Neste contexto, apresenta-se uma análise das condições de trabalho dos professores do ensino médio na pandemia, desenvolvendo em primeiro lugar uma caracterização crítica da dimensão socioeconômica e ambiental do território bonaerense. Em segundo lugar, analisa as políticas de continuidade pedagógica e a regulamentação do trabalho docente numa situação de excepcionalidade. Finalmente, e tendo em vista que a continuidade do ensino ocorreu num contexto de clara diluição das fronteiras entre o trabalho docente e a vida doméstica numa profissão altamente feminizada, destacam-se as principais tensões entre as condições sociossanitárias, a regulamentação do trabalho docente e as condições de gênero que afetam as dinâmicas que articulam o direito à educação e os direitos trabalhistas. A análise é o produto de um estudo em andamento, que pretende caracterizar as heterogeneidades territoriais e aprofundar sua incidência no trabalho dos professores através de fontes secundárias e uma pesquisa e entrevistas detalhadas em quatro localidades que expressam diferentes condições sociossanitárias. Palavras-chave: política educacional; trabalho docente; COVID-19; província de Buenos Aires.

Pedagogical continuity in Pandemic. A study on the intensification of teaching work in contexts of inequality

Abstract. The health emergency and the suspension of face-to-face classes make up a complex framework that in the province of Buenos Aires is worsened by its deep historical asymmetries.

Firstly, an analysis of the teaching work conditions in the pandemic in secondary education is presented, developing in the first place, a critical characterization of the socioeconomic and environmental dimension of the Buenos Aires territory. Secondly, the policies of pedagogical continuity and the regulation of teaching work in a situation of exceptionality are analysed. Finally, and because the maintenance of pedagogical continuity took place in a context of clear dilution of the limits between teaching work and domestic life in a highly feminized profession, the main tensions between socio-sanitary conditions, regulations on teaching work and gender conditions are pointed out and this affect the dynamics that articulate the right to education and labour rights. The analysis is the product of an ongoing study that proposes to characterize the territorial heterogeneities and investigate their impact on teaching work through secondary resources and a survey and in-depth interviews in four localities that express different socio-sanitary conditions.

Keywords: educational policy; teaching work; COVID-19; province of Buenos Aires. 


\section{Introducción}

A pocos días del inicio del ciclo lectivo 2020 en la República Argentina, el 11 de marzo de dicho año la Organización Mundial de la Salud (OMS) declaró el brote de la COVID-19 como pandemia. Un día después el Estado nacional argentino amplió la emergencia pública en materia sanitaria y desde el 20 de marzo, mediante el Decreto de Necesidad y Urgencia 297, dictaminó un “aislamiento social, preventivo y obligatorio" (ASPO) que implicó una amplia restricción para la realización de actividades sociales, educativas, laborales y económicas de manera presencial. Esta situación inédita para el sistema educativo en particular se prorrogó mediante sucesivos decretos durante prácticamente todo el ciclo lectivo 2020.

En ese escenario la escolarización, sin dudas, resultó uno de los ámbitos tempranamente afectados por la imposibilidad de sostener la distancia mínima requerida para garantizar las condiciones necesarias para evitar riesgos de contagio entre la comunidad educativa (entre otras medidas), debiendo las jurisdicciones suspender el trabajo escolar presencial y diseñar rápidamente respuestas de continuidad pedagógica a distancia.

En ese sentido el ASPO y las medidas adoptadas expusieron el problema del trabajo pedagógico a distancia en toda su magnitud y complejidad. Por lo cual, esta problemática en particular constituyó una oportunidad para indagar en profundidad el presente, pero también, frente a eventualidades próximas, el futuro de las condiciones de posibilidad garantizar el derecho a la educación en situaciones de no presencialidad.

Los profundos desafíos generados por la pandemia, cuya extensión en el tiempo e intensidad fueron mayores a los previstos, se expresan en las condiciones sociosanitarias de los territorios. Las dinámicas de las familias y la organización social del cuidado se vieron transformadas por la interrupción del cuidado institucionalizado de la infancia a través de las organizaciones escolares y la imposibilidad de acceder a otras redes de cuidado familiar. En el caso de los y las docentes, el acompañamiento escolar en sus respectivas familias se dio en simultáneo a la adecuación del trabajo docente y el sostenimiento del trabajo pedagógico.

Es en este marco donde nos interesó discutir la gestión política de la pandemia en el campo escolar, con foco en el nivel secundario, atendiendo a la heterogeneidad de las condiciones laborales docentes, reconfiguradas frente a la dilución de las fronteras entre trabajo y hogar; lo que significó la superposición de tareas docentes y de cuidado de personas a cargo y de las condiciones sociosanitarias y materiales de vida del hogar y las relativas al ámbito laboral.

Dado que se trata de una profesión altamente feminizada'; el artículo presenta una reflexión sobre las principales tensiones entre las condiciones sociosanitarias, las regulaciones del trabajo docente y las condiciones de género; que afectan las dinámicas que articulan el derecho a la educación y los derechos laborales en este escenario. El trabajo focaliza la mirada en la Provincia de Buenos Aires, la más poblada del país y donde las asimetrías y vulnerabilidades de la población son una deuda histórica.

\footnotetext{
${ }^{1}$ Un informe de 2016 elaborado por el Ministerio de Trabajo, Empleo y Seguridad Social de la Nación revela que el 73,6\% de la fuerza de trabajo ocupada en la enseñanza es femenina: https://www.argentina.gob.ar/sites/default/ files/informe_ctio_documentodetrabajo.pdf
} 
Las reflexiones aquí vertidas son producto del proyecto de investigación "Condiciones y condicionantes del trabajo docente en el nivel secundario en contextos de pandemia. De la presencialidad a la virtualidad obligada", financiado por el Programa de Articulación y Fortalecimiento Federal de las Capacidades en Ciencia y Tecnología COVID-19 del Ministerio de Ciencia, Tecnología e Innovación, específicamente destinado a producir conocimientos sobre áreas estratégicas en el marco de la pandemia.

\section{Metodología}

El artículo recupera los resultados parciales de una investigación en curso que analiza condiciones del trabajo docente en el contexto de la pandemia y que cuenta con financiamiento específico del Ministerio de Ciencia y Técnica de la Nación. Se trata de una investigación cuali-cuantitativa, que se propuso analizar los procesos de acompañamiento pedagógico en el marco de la suspensión de clases presenciales y el retorno a las aulas a partir del estudio de las condiciones y condicionantes del trabajo docente en contextos de excepcionalidad en el nivel secundario común de la Provincia de Buenos Aires.

Para este artículo, primero se relevaron y analizaron fuentes secundarias sistematizadas en el censo de población hogares y vivienda y en estadísticas propias de la provincia que permiten dar cuenta de la heterogeneidad y vulnerabilidad sociosanitaria. Para la vulnerabilidad se tomaron 3 indicadores que permitieron relevar, en alguna medida, esta condición: acceso al agua de red, disponibilidad de desagüe cloacal y nivel de Necesidades Básicas Insatisfechas (NBI). Los 135 municipios fueron clasificados en 4 grupos de acuerdo a dichos indicadores: dentro del Grupo 1 de baja vulnerabilidad, incluye a municipios que muestran una provisión de agua de red superior al $80 \%$, desagüe cloacal de más del $50 \%$ y un nivel de NBI inferior al $5 \%$ de la población. El Grupo 2 media comprende, cobertura de agua de entre 50\% y 80\%, desagüe cloacal entre $25 \%$ y $50 \%$ y nivel de NBI entre $5 \%$ y $10 \%$. En el grupo 3 alta vulnerabilidad se incluyen los municipios que tienen un indicador medio o alto en alguno de los indicadores trabajados. Por último, en el Grupo 4 se agrupan a municipios en situación crítica con agua de red inferior al 50\%, desagüe cloacal menor al 25\% y nivel de NBI por encima del $10 \%$ de la población. La unidad de medida utilizada en todos los casos son los hogares. Los datos se basan en el CENSO 2010 del INDEC.

En segundo lugar se recuperaron los resultados de una encuesta autoadministrada, dirigida a docentes del nivel secundario común, que trabajan en cuatro distritos de la provincia de Buenos Aires, que son representativos de la heterogeneidad del territorio bonaerense: el municipio de General Pueyrredón (G1), Baradero (G2), Tigre (G3) y Moreno (G4). El relevamiento se implementó entre octubre y noviembre de 2020, contó con la participación de 1531 docentes, complementa la información producida por organismos públicos y organizaciones sindicales sobre condiciones de trabajo docente, y aporta especificidad local que hasta el momento no se ha producido o desagregado. La encuesta relevó información relacionada a componentes del hogar, características de las viviendas, acceso a dispositivos tecnológicos, conexión a internet, ingresos, trabajo no remunerado, trabajo pedagógico, entre otras. 


\section{La provincia de Buenos Aires y los desafíos de la gestión de la heterogeneidad en el contexto de la pandemia}

Las desigualdades socioeducativas que se observan en el territorio argentino atraviesan con especial atención a la provincia de Buenos Aires, la más extensa del territorio continental argentino (sin considerar la Antártida Argentina e Islas del Atlántico Sur). La población bonaerense representaba el 38,95 \% de la población total del país, unos 15.625 .084 habitantes radicados en centros urbanos, con proyecciones a 2020 de 17.541 .141 habitantes. La provincia gestiona el sistema educativo más "complejo del país y uno de los más vastos de América Latina" (Agoff, Bertranou y Foglia, 2016, p.123). Algunos datos que lo evidencian señalan que concentraba, en 2019, a 20.680 unidades educativas con 5.216.192 de alumnos distribuidos en gestión estatal (3.684.568) y privada (1.531.624), de los cuales 2.205.706 asistían a establecimiento en el interior bonaerense y 3.010 .486 en el Conurbano Bonaerense (Dirección de Información y Estadística, 2019).

La provincia se organiza en 135 partidos, que son jurisdicciones político- administrativas de segundo orden y coinciden con los municipios. Se observa la coexistencia de dos regiones geográficas diferenciadas con dinámica demográfica propia: el Gran Buenos Aires (GBA) o conurbano bonaerense, integrado por 24 partidos que rodean a la Ciudad Autónoma de Buenos Aires (CABA) que concentra al 63,46\% de la población, y por otro, el interior y la capital provincial, conformado por 111 partidos dónde se localiza el resto de la población (INDEC, 2010).

Las dos áreas experimentaron distintos procesos de asentamiento de la población que le imprimen una singularidad propia: por un lado, el interior provincial con pueblos y ciudades cuyo asentamiento data del siglo XIX; y por otro la conurbación, que, junto a la CABA, forma parte de la metrópolis denominada AMBA y que creció de forma notoria al calor de la denominada "Industrialización Sustitutiva de Importaciones". Aunque ambas áreas comparten patrones de asentamiento poblacional y productivo similares a su interior, no son homogéneas, sobre todo en lo que concierne a la disponibilidad de acceso a los servicios básicos, por eso sostenemos que son dos provincias. Asimismo, fue el territorio más afectado por la expansión de la COVID 19.

Los municipios del Interior sin lugar a dudas cuentan con mayor cobertura de los servicios de infraestructura por redes y también mayor cobertura médica provista por las obras sociales en relación a lo que sucede con los municipios del Conurbano (Provincia de Buenos Aires, 2015).

La evolución de los principales indicadores laborales de la provincia (salario real y desocupación) muestran indicios de un proceso de deterioro generalizado que, por el peso específico de Buenos Aires, repercuten a nivel nacional. A partir de 2015 se puso fin a un ciclo de creación de empleo iniciado en 2002, que, con distintas coyunturas, llegó a una tasa de desocupación que se ubicó en un 5,9\% a nivel nacional y en $6,7 \%$ en la provincia. Entre 2016 y 2018 comienza un ciclo en el que se eleva el nivel de desempleo, que llega al $11,8 \%$ en la provincia y a $9,6 \%$ a nivel nacional. Estos indicadores, se incrementaron durante 2019 y también en 2020 donde alcanzaron el $13,1 \%$ a nivel nacional y el $13,8 \%$ dentro de los partidos del Gran Buenos Aires, con una caída de la actividad que se ubicó en 37,6 (INDEC, 2020). 
Los indicadores de condiciones de vida que muestra la provincia de Buenos Aires muestran una situación general que es más grave de la que se registra a nivel nacional. En términos de pobreza por ingresos, la provincia de Buenos Aires tiene, para el primer semestre de 2020, un 46,1\% de personas en condición de pobreza de los cuales un $13 \%$ son indigentes (DPE, 2020) mientras que, a nivel nacional, los indicadores son de 40,9\% 10,5\% respectivamente (INDEC, 2020). Esta situación expone a situaciones de clara vulnerabilidad a la población y afectan derechos humanos inalienables como la salud, las condiciones de vida, el trabajo y la educación.

Hay una clara desigualdad en la distribución de los ingresos en suelo bonaerense sea per cápita individual como familiar. Durante el primer semestre de 2020 el $40 \%$ de la población bonaerense con menores ingresos (aquellos que se ubican en los deciles 1 a 4) se apropian del 14\%, y los de mayores ingresos (deciles 9 y 10) del 48\%. Esta situación ubicaba a las mujeres en peores condiciones cuando se observa a nivel de la escala individual. Así las mujeres en los deciles 1 a 4 participaban del $24,2 \%$ y los varones del $15,8 \%$, lo que se revertía en los mayores deciles donde las mujeres tienen menos participación que los varones, 6,4\% y 12,6\%. Esta desigualdad también se observaba en los ingresos medios en las mujeres era de \$26.259 y los varones de $\$ 34.979$ (DPE, 2020). La desigualdad entre los sexos se extiende también a los valores medios: el ingreso individual promedio femenino es de \$26.259 y el de los varones de $\$ 34.979$, marcando una diferencia de $\$ 8.720$. Si se observa el componente hogares los de menores ingresos se apropian del $16,5 \%$ y los de los deciles más altos del 44,1\%.

En síntesis, la información disponible evidencia que la provincia de Buenos Aires enfrenta heterogeneidades estructurales en lo que refiere a distribución de ingresos, de recursos y de condiciones de vida en general entre distintos sectores sociales, pero también se observan intra municipios, en cada una de las regiones; y por género. No menos fue el impacto subjetivo ASPO sobre estas condiciones de base: se intensificaron la preocupación sobre la discontinuidad de los ingresos laborales de los hogares, en especial por el nivel de cuentapropismo, las dificultades para acceder a los alimentos y artículos de limpieza dado la falta de ingresos o problemas de logística en la reposición, entre otras preocupaciones (Maceira et al., 2020).

Con el objetivo de poder dar cuenta de la heterogeneidad que presenta el vasto territorio bonaerense en lo que respecta a las condiciones sociosanitarias de la población directamente vinculadas con las condiciones de cuidado frente a la pandemia, elaboramos una clasificación de los municipios en grupos según su nivel de vulnerabilidad social según se explicita en el apartado metodológico.

En términos generales, la provincia de Buenos Aires tiene la mayor parte de sus municipios comprendidos dentro del Grupo 2: se trata de un total de 91 distritos que representan casi el $67 \%$ del total. Si nos basamos en estas referencias, podría parecer que la parte de la población que se encuentra en situación de vulnerabilidad crítica en la provincia de Buenos Aires es poco significativa en términos relativos, dado que menos del $10 \%$ de los distritos se clasifica dentro del Grupo 4. Sin embargo, se trata de municipios muy poblados que superan las 150.000 personas en cada caso. Así, si se suma la población de todos estos partidos (Almirante Brown, Escobar, Ezeiza, José C. Paz, Malvinas Argentinas, Merlo, Moreno y Pilar) se llega a una cifra que supera 
los 2.700 .000 personas, lo que representa casi el $18 \%$ del total de la población de la provincia. La situación de vulnerabilidad crítica tiene así una incidencia considerable en términos absolutos y relativos dentro del territorio provincial: uno de cada 5 hogares se encuentra en condición de vulnerabilidad crítica, especialmente dentro del GBA. Esto implica que una cuarta parte de los distritos que conforman el conurbano bonaerense se encuentra en condición de vulnerabilidad crítica. En términos poblacionales, estos municipios suman un total de más de 2 millones de personas, lo que representa poco más del 23\% del total de habitantes del GBA. El grueso de la población bonaerense se caracteriza por lo que podemos considerar como un nivel moderado de vulnerabilidad social. La falta de acceso al agua conforma una deuda pendiente en territorio bonaerense, que más allá de contar con distintos prestadores del servicio está lejos de abastecer a la población, especialmente urbana. En el marco de la pandemia esta situación ha recrudecido las condiciones de vida y sanitarias.

\section{Escolarización y trabajo docente: las políticas educativas frente a la pandemia}

Las desigualdades estructurales recién reseñadas en la sección anterior, en su mayoría han variado poco a lo largo de la última década y nos posicionan frente a desafíos peculiares en el campo escolar, que además no sólo acompaña esta fragmentación sino que asume su propia dinámica interna en el nivel de educación secundaria en términos de infraestructura, oferta curricular y alcanza al propio cuerpo docente, sus condiciones de vida, su capital cultural, trayectorias laborales y estilos pedagógicos (Poliak, 2009; Birgin y Charovsky, 2013; Gluz y Rodríguez, 2015) e impactan particularmente en algunos territorios, como el Conurbano Bonaerense (Southwell, 2015).

Se trata de un nivel que además, viene transitando diversas reformas que desde los ‘90 que han dislocado su identidad, incorporando y redefiniendo los saberes docentes por el cambio de estructura primero, luego por los cambios curriculares, que si bien se llevaron adelante sin vulnerar derechos laborales adquiridos, ha significado en algunos casos una compleja articulación entre perfiles docentes y asignaturas (Gluz y Rodríguez, 2015).

El tipo de inserción laboral, que está mayormente organizado en torno a horasmódulo frente a curso -salvo algunas experiencias piloto-, así como la carga horaria de las asignaturas, deriva en algunos casos en la multiplicación de instituciones de trabajo; y en todos los casos, en la diversidad de cursos y, por ende, en una importante cantidad de estudiantes a cargo. Esta situación se constituye sin dudas en un desafío para el desarrollo de estrategias de continuidad pedagógica enmarcadas en las recomendaciones del "sostenimiento del lazo" que surgieron tanto desde la cartera educativa nacional como provincial y agrega a la heterogeneidad común a otros niveles, la vinculada a las modalidades, ciclos formativos y áreas disciplinares; así como la variabilidad de cursos a cargo de cada docente.

Estas condiciones de trabajo docente (Martínez, 1999) han sido dislocadas en el contexto de la pandemia, por su incidencia en la toma de decisiones sobre enseñanza y sostén del vínculo con las y los estudiantes en el marco de la continuidad pedagógica; y por sus efectos sobre el malestar docente (Martinez, Collazo y Liss, 2009). Por un 
lado, las regulaciones existentes resultaron inadecuadas frente a la excepcionalidad, lo que requirió la reorganización del andamiaje normativo; para atender centralmente a una dimensión que ha sido especialmente alterada por la suspensión de las clases presenciales que constituyen los escenarios para hacer referencia al contexto espaciotemporal de la representación, pero también a las significaciones subjetivas con que se dota ese contexto (Martínez, 1999). Estos escenarios ligados a la gramática de la escolaridad, a los mecanismos de control institucional y a las culturas escolares que allí se construyen, se articulan con las desigualdades en los territorios, que se profundizan incluso al interior de los barrios y que constituyen un factor ineludible de diferenciación del trabajo docente, de los desafíos que afrontan frente a escuelas que portan distintos proyectos sociales y culturales y atienden poblaciones atravesadas por problemáticas específicas. Estos desafíos se intensifican en el contexto de la pandemia respecto a variables como la conectividad, las condiciones sociales, materiales y culturales del hogar para construir soportes para la continuidad pedagógica y la heterogeneidad de condiciones sociosanitarias críticas para garantizar el cuidado.

En este marco, las políticas educativas gestionaron la pandemia en lo que podrían diferenciarse como tres etapas. Una primera etapa donde las decisiones se concentraron en medidas preventivas que fueron previas al dictamen del ASPO, que tuvieron el propósito fundamental de evitar la propagación del virus. En este momento inicial, que tuvo lugar cuando empezaron a registrarse los primeros contagios y en el que comenzaba a preverse un escenario que anticipaba la posibilidad del ASPO (y que continuó en las primeras semanas de la implementación del mismo), las medidas tuvieron un carácter centralmente sanitario en relación a la necesidad de brindar respuestas urgentes para la problemática que se acercaba. Tanto desde el gobierno nacional como desde el gobierno bonaerense se implementaron políticas y mecanismos organizativos para reglamentar la utilización de licencias extraordinarias por parte de aquellas personas que regresaron de un viaje por países con circulación comunitaria del virus, así como determinar el cierre de aquellas instituciones educativas en las que se confirmara un caso positivo.

El 15 de marzo el gobierno nacional determinó la suspensión de clases presenciales por quince días corridos a partir del 16 de marzo de 2020 y fue refrendada por la Provincia de Buenos Aires mediante la Resolución 554 de la DGCyE de igual fecha. A partir de esta norma aparecen en escena dos dimensiones que vertebrarán la segunda etapa y que tienen implicancias directas sobre el trabajo docente: el sostenimiento de la continuidad pedagógica y la atención a la vulnerabilidad: "durante el plazo que dure la suspensión de asistencia de estudiantes, el personal docente, no docente y directivo concurrirá normalmente a los efectos de mantener el desarrollo habitual de las actividades administrativas, la coordinación de los servicios sociales y las actividades pedagógicas que se programen para el presente período de excepcionalidad". Se insta entonces a la conducción de los establecimientos educativos a que organicen la disponibilidad y los perfiles del personal docente y auxiliar que cumple actividades presenciales en los establecimientos educativos a fin de asegurar fundamentalmente la plena implementación del programa de continuidad pedagógica y la prestación del Servicio Alimentario Escolar (SAE). Ello implicó adicionalmente regimentar nuevas licencias para personas incluidas en las categorías de riesgo -mayores de 60 años, 
embarazadas, enfermedades crónicas, entre otras- como también para trabajadorxs con menores a cargo, al tiempo que estableció la continuidad del SAE bajo la modalidad de bolsones de emergencia, por lo que reguló la apertura de forma quincenal de las instituciones que brindaron este soporte alimentario, lo cual supuso para las instituciones una reorganización del cuerpo docente para cumplir con esta tarea. ${ }^{2}$

El dictamen del ASPO inicia la segunda etapa, cambia el foco al concentrarse prioritariamente en las alternativas para el sostenimiento de la continuidad pedagógica en el contexto de la suspensión de las clases presenciales, lo que implicó desplegar una serie de propuestas inéditas que alteraron radicalmente el trabajo docente. En esta etapa las políticas públicas se van a estructurar en torno a los dos ejes fundamentales que mencionamos anteriormente: el sostenimiento de la continuidad pedagógica y la atención a la vulnerabilidad. Este segundo momento se caracterizó por un fuerte dinamismo propio también del desarrollo de la pandemia, el hecho de que la continuidad del ASPO fuera prorrogada por el gobierno nacional quincenalmente, tuvo implicaciones para todas las decisiones en materia educativa tanto para el gobierno nacional como provincial. En este sentido, fueron surgiendo una serie de problemáticas en la medida que se extendía el aislamiento, y que exigieron nuevas respuestas desde las gestiones.

Respecto al sostenimiento de la continuidad pedagógica, tanto desde el gobierno nacional como el provincial el acento estuvo puesto inicialmente en el acceso a los contenidos educativos. Entre las medidas más relevantes cabe mencionar el lanzamiento de los portales Seguimos educando ${ }^{3}$ y Continuemos estudiando 4 que tuvieron el propósito de garantizar el acceso a recursos y actividades educativas para todas/ os las y los docentes de todos los niveles ${ }^{5}$. Del mismo modo, en el marco de ambos programas se editaron y distribuyeron una serie de cuadernillos que fueron llegando a las escuelas en tiempos distintos, priorizando en un primer momento las asignaturas troncales de la secundaria y luego se fueron incorporando el resto de las materias en tanto continuaba el ASPO. Ambas gestiones evidenciaron de este modo una rápida respuesta en términos de construir soportes para el trabajo pedagógico: Seguimos Educando fue lanzado el 16 de marzo, y Continuemos estudiando el 19 de marzo. La Provincia de Buenos Aires a su vez, también generó una política de apoyo técnicopedagógico a las/los docentes con la facilitación de aulas virtuales disponibles para el encuentro con estudiantes y el dictado de clases a requerimiento de docentes, como también la asistencia y apoyo técnico a equipos de conducción y equipos docentes desde los CIIES con espacios de formación permanente y acompañamiento para el uso de herramientas educativas en el marco de la virtualidad.

\footnotetext{
${ }^{2}$ Es importante señalar que a partir de la Res. 657 (22 de marzo), las tareas de continuidad pedagógica quedan exceptuadas del ASPO y, por otro lado, que es recién a partir del 29 de octubre que se encuadran las tareas docentes en contexto de no presencialidad como resultado de las negociaciones de la Mesa Técnica Paritaria de Condiciones Laborales. En el pliego de reconocimientos de derechos laborales, las tareas de cuidado (menores a cargo) no aparecen como parte de los principales acuerdos paritarios.

${ }^{3}$ El Programa fue creado conjuntamente entre el Ministerio de Educación de la Nación, Educar S.A. y Contenidos Públicos S.A., incluye también la producción y emisión de la programación audiovisual a través de las señales dependientes de la órbita de la Secretaría de Medios y Comunicación Pública: Televisión Pública Argentina y sus repetidoras, Encuentro, Paka Paka, DeporTV, Radio Nacional y Cont.ar.

${ }^{4}$ El portal Continuemos Estudiando fue desarrollado por los equipos de Tecnología Educativa de la DGCyE.

${ }^{5}$ Ambos portales son de navegación gratuita.
} 
Frente a la inesperada extensión del ASPO, las preocupaciones en torno a la continuidad pedagógica fueron virando hacia la posibilidad de dar respuestas más definitivas vinculadas por un lado al sostenimiento del vínculo pedagógico y por el otro, la problemática de la reorganización de los contenidos y la evaluación y acreditación.

Respecto al primero de los ejes mencionados, la atención a la vulnerabilidad cobró mayor dimensión en esta segunda etapa. Si bien en un inicio estuvo concentrada en garantizar la continuidad del SAE en diversas formas (comedores y entrega de bolsones de comida) y el acceso a través de las distintas medidas vinculadas a la gratuidad de portales web, entrega de netbooks, materiales impresos, etc., de los que ya hemos hecho referencia, la provincia de Buenos Aires tomó una serie de medidas en relación a brindar "algunas orientaciones para el sostenimiento del vínculo pedagógico, como reconocer los tiempos heterogéneos de aprendizaje y el significado de la escuela en la conformación de un colectivo, especialmente en el ASPO" y "la importancia de seguir profundizando los vínculos pedagógicos con los y las estudiantes, sosteniendo una mirada de cuidado".

La creación de los Equipos Focales Territoriales Educativos para la Emergencia (EFTEE) también apuntó a la atención de las trayectorias. A partir de este dispositivo se instrumenta administrativamente la convocatoria de las/los docentes que integren Equipos de Orientación Escolar o que ocupen algún puesto de trabajo docente en los Centros Educativos Complementarios y se detalla el procedimiento de selección de los cargos para la conformación de dichos equipos. Los mismos, son entendidos como una medida extraordinaria del contexto, y señalan una función específica del trabajo docente abocada al sostenimiento de vínculos pedagógicos en el marco del ASPO y a la atención de situaciones conflictivas. Estas normas señalan la necesidad de que las/ los docentes presten atención a las trayectorias reales aún con más cuidado que en el trabajo presencial, y ponen el acento en la evaluación en relación con el fortalecimiento de las decisiones pedagógicas y didácticas adoptadas para mejorar esas trayectorias. Resulta importante señalar que todas estas políticas conllevan de una resignificación del trabajo docente en esta coyuntura, que apuesta al trabajo interdisciplinario con fuerte anclaje territorial considerando las heterogeneidades de la provincia.

El 15 de mayo el Consejo Federal de Educación (CFE), aprobó las “Orientaciones para los procesos de evaluación en el marco de la continuidad pedagógica" que indicaba que ningún alumno del país será calificado mientras las clases presenciales se encuentren suspendidas, la priorización y reorganización de los contenidos, y establece que la acreditación del nivel podría tener lugar entre finales de 2020 y la primera mitad de marzo de 2021. La provincia de Buenos Aires en línea con la misma aprueba los lineamientos de Evaluación, Acreditación y Promoción donde además explicita la necesidad de prever un abordaje específico para acompañar las trayectorias educativas interniveles y de los tramos de inicio y finalización de los niveles de enseñanza, además de anticipar la elaboración de material de trabajo y orientación para docentes.

Las decisiones en materia de reorganización de la enseñanza surgieron también ante las redefiniciones en el calendario escolar debido a la expansión delASPO. EI CFE resuelve avanzar en la reorganización curricular a través de la llamada Priorización de contenidos entendida como "(...) un proceso de reorganización de los contenidos y metas de aprendizaje previstos en normativas curriculares vigentes en las jurisdic- 
ciones, para los ciclos, niveles y modalidades de la educación obligatoria", dejando en manos de las jurisdicciones la implementación de la misma. En esta normativa, se explicita que esta organización de la enseñanza deberá considerar "el diseño de las acciones que se definan para el avance progresivo de presencialidad -integración pedagógica de tiempos de presencialidad y de no presencialidad-, mediante la explicitación de los itinerarios de enlace entre ambas formas de escolarización", cuestión que se retomará en la tercera etapa.

En paralelo, una acción de relevancia desplegada por la provincia se vinculó a la garantía de los ingresos salariales docentes. Dada la suspensión de los actos públicos presenciales y la consecuente falta de cobertura de cargos en la Provincia de Buenos Aires, se crea el Programa PIEDAS (Programa de Incorporación Especial de Docentes y Auxiliares Suplentes), destinado a trabajadores y trabajadoras docentes y a auxiliares enmarcados/as en regímenes de suplencias, que no hubieran podido acceder a cargos por la imposibilidad de realizar actos públicos. De esta forma se cubrieron los cargos en las instituciones escolares para realizar las actividades extraordinarias exigidas por la emergencia sanitaria, al tiempo que se dio respuesta a los reclamos por continuidad laboral de los y las docentes de toda la Provincia. Recién el día 11 de agosto a través del Decreto 690 de la Gobernación de la Provincia, se aprueban y anuncian los Actos Públicos Digitales para la adaptación de la publicación de las vacantes, la inscripción, notificación y toma de posesión de los cargos docentes a través de medios tecnológicos, para evitar aglomeraciones de más de diez personas en lugares cerrados

La tercera etapa tendrá como foco el retorno a la presencialidad y la revinculación con estudiantes sin continuidad pedagógica. Hacia los meses de junio y principios de julio se inició la discusión sobre la vuelta a la presencialidad. Al respecto, el 2 de julio el CFE hace público el Protocolo Marco y Lineamientos Federales para el Retorno a Clases Presenciales en la Educación Obligatoria y en los Institutos Superiores. Y unos meses después, el 01 de septiembre, lanza el Programa ACOMPAÑAR: Puentes de Igualdad cuyo principal objetivo es "promover y facilitar la reanudación de trayectorias escolares y educativas cuya interrupción ha sido potenciada por la pandemia y su secuela de desigualdades", previendo la articulación con cada jurisdicción provincial.

En torno a la revinculación, la provincia lanza el Programa "Acompañamiento a las Trayectorias y Revinculación" (ATR) como estrategia para la revinculación y acompañamiento pedagógico de las y los estudiantes que hayan visto vulneradas sus trayectorias educativas con el consecuente impacto que ello genera en el proceso de continuidad pedagógica. El programa propone un dispositivo de visitas domiciliarias, acompañamiento y acciones de revinculación con la escolaridad obligatoria de las/ los estudiantes que hayan discontinuado su contacto con los establecimientos. Convoca a estudiantes avanzados de carreras docentes y docentes suplentes (a partir del desarrollo de experiencias de prácticas de enseñanza contextualizadas) para el despliegue de estas tareas. En el caso de aquellos/las docentes en formación, se despliegan apoyos institucionales para la tarea por parte de docentes de los Institutos de Formación Docente. 
La cuestión sociosanitaria tendrá una relevancia específica en la Provincia para el regreso progresivo a las clases presenciales en los distritos evaluados de "bajo riesgo epidemiológico". El "regreso seguro a las aulas" a partir del 26 de octubre se distingue del protocolo nacional aprobado en el CFE, por una minuciosa regulación en términos sociosanitarios. El protocolo señala que las escuelas deben "disponer de agua para la limpieza y de agua potable para consumo humano, así como deberán contar con la infraestructura sanitaria adecuada", una cuestión difícil de cumplir en una jurisdicción de deudas históricas en esta esta cuestión tal como se mencionó anteriormente. Asimismo, la distancia mínima requerida entre personas será más compleja de cumplir en los territorios de mayor hacinamiento donde la matrícula es más elevada, lo que dificultará la vuelta a clases en territorios de alta vulnerabilidad, situación que el programa ATR puede atenuar, pero no sortear (además de requerir su continuidad para el ciclo lectivo 2021). Es de destacar el reconocimiento de las dificultades para el trabajo colectivo docente, y la estipulación en función de ello de destinar antes del inicio, de "dos semanas a la planificación de la continuidad pedagógica según los contenidos curriculares priorizados por cada nivel y modalidad; a la organización de la enseñanza en formatos bimodales (clases presenciales y no presenciales) de aceleración, pluriaño, entre otros, y al desarrollo de espacios institucionales de formación y sistematización de lo trabajado; así como también a la capacitación de equipos de dirección, docentes y auxiliares en medidas de bioseguridad obligatorias para las escuelas y las y los estudiantes"

\section{Profesoras, madres y jefas de hogar. Las políticas públicas educativas frente a las cuestiones de género}

La pandemia encontró al cuerpo docente en una situación específica que puede definirse tanto en términos de ventajas, como de desventajas. Por un lado, se trata de una población que, como otros trabajadores formales, por el carácter protegido de su inserción laboral no se vieron comprometidos sus ingresos (Birgin, 1999) que a diferencia de la desprotección que afecta a la población en contextos de crisis (Kessler y Assusa, 2020), sostuvieron ingresos y redes. Pero, por otro lado, se vieron sometidos a la reconfiguración de su trabajo sin contar con experiencias, sin los recursos materiales para sostenerlo en el hogar, sin recursos pedagógicos extendidos para el nuevo formato y con nuevas y mayores demandas para la transmisión. A ello se suma la especificidad del trabajo docente en el nivel secundario y la condición altamente feminizada del profesorado que configuró desafíos significativos para el sostenimiento de la continuidad pedagógica.

Las políticas adoptadas se dirigieron a la totalidad de las y los docentes, sin gran consideración excepto al inicio, y no de forma generalizada, donde se asignaron licencias a docentes con personas a cargo. La pandemia ha agravado las desigualdades de género por el incremento de la pobreza, el desempleo y la mayor sobrecarga de trabajo doméstico y de cuidados no remunerado. Cabe recordar que el cuidado es provisto por la familia, el Estado, el mercado y la comunidad (Razavi, 2007). No obstante, el trabajo no remunerado al interior de los hogares y en especial, el cuidado en la primera infancia es la fuente central del cuidado asumido, centralmente por mujeres (Rodríguez, 2010). Esto último cobró vital importancia en tanto las decisiones incidieron en la organización social del cuidado, ya que se suspendieron las 
clases presenciales en escuelas y centros de primera infancia, en simultáneo con la imposibilidad de organizar el cuidado con trabajo doméstico remunerado y las redes familiares no convivientes (Arza, 2020). En ese contexto complejo la organización del cuidado de niños, niñas y adolescentes, así como adultos mayores y otras personas con problemas de salud en los hogares tuvieron que combinarse con el desarrollo del trabajo docente.

Las condiciones y los condicionantes para sostener la continuidad pedagógica pone de relieve importantes brechas vinculadas a las condiciones de género, a la desigual responsabilidad frente a las tareas de cuidado y de trabajo doméstico y el modo como ello afecta la disponibilidad de tiempo para la actividad de enseñanza. Los primeros relevamientos dieron cuenta tempranamente de estas dificultades. En la Evaluación Nacional del Proceso de Continuidad Pedagógica ${ }^{6}$ en la que se analizan las dificultades de los y las docentes para desarrollar su trabajo, si bien las dificultades de conectividad y tecnologías de comunicación son las razones principales de esta dimensión, también se menciona que un poco más del tercio de los y las docentes declaran tener problemas para compatibilizar las tareas pedagógicas con las tareas de cuidad (36\%). dicho porcentaje, además ascienden considerablemente en el caso de los y las docentes de secundaria, donde asciende al 41,5\% de las y los encuestados (SEIE-ME, 2020, p. 13). Informaciones similares relevan las encuestas desarrolladas por los sindicatos docentes.

En el caso de la encuesta de CTERA, Salud y condiciones de trabajo docente en tiempos de emergencia sanitaria COVID19, vale la pena resaltar que el $84 \%$ de los 15.634 docentes de las 24 provincias que participaron son mujeres, y en el $65 \%$ de los hogares los y las docentes conviven con niños/as y adolescentes que tienen a su cargo. De acuerdo al procesamiento por géneros que realiza CTERA sobre este punto, es posible afirmar que, "para las mujeres (86\%) la convivencia con las tareas domésticas y familiares está resultando más problemática que para los varones (79\%)" (CTERA, 2020, p. 26). Adicionalmente, se plantea que "mientras que el 32\% de las mujeres con niñas/os adolescentes a cargo no convive con otra persona adulta con quien comparta las tareas de cuidado, sólo el 15\% de los varones con niñas/os adolescentes a cargo se encuentra en esa situación. " (CTERA, 2020, p. 25). En relación a las tareas de cuidados de adultos mayores o de personas que forman parte de grupos de riesgo, el 65\% declara tener responsabilidades de cuidados directos, con más peso en las mujeres (66\% frente al 59\% en los varones) (CTERA, 2020, p. 27). Por otro lado, y a diferencia del informe ministerial, la "convivencia del trabajo con las tareas y demandas domésticas y familiares" se consigna como la segunda problemática laboral en contexto de emergencia sanitaria en relación con las otras problemáticas indicadas (conectividad, contactos con estudiantes e insuficiencia del salario). En sintonía con esta información, el relevamiento desarrollado específicamente en la Provincia de Buenos Aires por el SUTEBA, Encuesta Provincial de Trabajo Docente en Contexto de Aislamiento Obligatorio ${ }^{7}$ da cuenta de la "Carga de trabajo de cuidado de otras

\footnotetext{
${ }^{6}$ Encuesta desarrollada por la Secretaría de Evaluación e Información Educativa del Ministerio de Educación de la Nación. La misma se implementó en junio 2020 e incluye relevamientos de hogares, equipos directivos y docentes. Participaron más de 21 mil docentes de todo el país.

${ }^{7}$ La encuesta se desarrolló durante la segunda quincena de mayo de 2020 y participaron 5038 docentes de toda la provincia de Buenos Aires. Se presupone que los/las encuestados/as pertenecen a instituciones de gestión estatal, aunque no se aclara en el informe correspondiente.
} 
personas en el hogar", de los que se desprende que entre casi el 50 y el $60 \%$ de los y las docentes comparten las tareas de cuidado con otra persona adulta. El primer informe no presenta datos desagregados por género que permitan profundizar en la distribución de estas tareas. Aunque no sólo se ven condicionadas/os en su trabajo por el peso de las tareas de cuidado. Adicionalmente, las condiciones del hogar no siempre resultan propias. Según el relevamiento del SUTEBA, sólo el 34\% de los y la docentes encuestados/as cuenta con un espacio tranquilo para trabajar dentro de su hogar de manera permanente. Esta última dimensión cambia drásticamente entre aquellos docentes que cuentan con niños/as y/o adolescentes en el hogar. Mientras que en aquellos hogares en los que los/las docentes no conviven con niños/as y/o adolescente el porcentaje de espacios tranquilos de trabajo de manera permanente asciende a $56,9 \%$, en los hogares que sí cuentan con ellos, desciende prácticamente a la mitad $(23,6 \%)$.

En diversos grados de especificidad, estos relevamientos muestran que las tareas de cuidado y el trabajo doméstico aparecen como problemáticas que afectan considerablemente el desarrollo de las tareas de enseñanza, y que dichas tareas afectan mayormente a las mujeres.

Considerando esta problemática, los primeros datos de la investigación relevados a través de una encuesta en cuatro distritos a profesores/as sólo de nivel secundario estatal presenta tendencias acordes a las nacionales y provinciales, como la presencia en un $52 \%$ de hogares con menores a cargo y la participación mayoritaria, en términos absolutos, de las mujeres con familiares a cargo en el 51,3\% del total de encuestados/ as. La distribución por géneros de las tareas de cuidado, arroja que las mujeres asumen en una proporción mayor este trabajo $(65,4 \%)$ que los varones (60\%) que participaron de la encuesta. Asimismo, durante el ASPO para el $46 \%$ de las mujeres aumentó la carga horaria de tareas de cuidado, con un impacto algo menor en los varones donde fue del $42 \%$. Es de destacar, no obstante, que estos aumentos suceden frente a puntos de partida asimétricos: mientras anteriormente al ASPO los varones destinan mayormente entre $1-2$ horas (52\%) y entre 3 y 5 (34\%); las mujeres dedicaban más tiempo en el rango de mayor cantidad de horas (33 y 51\% respectivamente)

Una dinámica similar sucede en relación al aumento de horas de trabajo doméstico a partir del ASPO en aquellas/os docentes que tienen personas a cargo, que asciende entre el $42 \%$ (varones) y el $45 \%$ (mujeres) según género para el total de la muestra. Complementariamente, si bien el procesamiento general de la cantidad de horas de estas tareas, da cuenta que para el $40 \%$ de ambos géneros aumentaron las horas de trabajo doméstico, el porcentaje de los casos en que se mantuvo la misma dedicación de horas expresa diferencias importantes por género. Así, mientras para las mujeres representa un $36 \%$, para los varones es de un $46 \%$, por lo cual se infiere que estos últimos vieron menos alterada su dedicación a este tipo de tareas durante el ASPO.

Es posible sostener que el trabajo para las mujeres se intensifica en múltiples sentidos: por las demandas de trabajo docente, por las demandas de tareas de cuidado, por el acompañamiento a la escolarización de sus propios hijos y por el incremento del trabajo doméstico no remunerado. Esto se acentúa en los territorios de mayor vulnerabilidad (los grupos 3 y 4 tienen al 52 y $54 \%$ de las mujeres con personas a cargo, 
lo que se reduce al $45 \%$ en el grupo 1 de vulnerabilidad) y en territorios de mayor ruralidad. De este modo se evidencian más tareas, extendiendo la jornada escolar a la vez que un deterioro de las condiciones de ejercicio de las tareas de enseñar y una mayor necesidad de formación que va en detrimento del tiempo disponible para ello (Hargreaves, 1998).

Finalmente, 26,6\% de los docentes indica que ha incrementado el nivel de conflictividad entre miembros del hogar durante el ASPO, lo que en un contexto donde además la violencia sobre las mujeres ha alcanzado niveles significativos, amerita un análisis de mayor profundización en el desarrollo de la investigación.

\section{A modo de cierre: tensiones entre condiciones sociosanitarias, condiciones de trabajo docente y políticas de cuidado en el territorio bonaerense}

El sistema educativo bonaerense ha mostrado una importante capacidad de adaptación a los cambios en tiempos récord, desmitificando cualquier supuesto respecto de las estructuras anquilosadas incapaces de adaptación a las circunstancias.

Ahora bien, estas vertiginosas transformaciones hacen necesario integrar a las dimensiones históricamente consideradas para el estudio de la estructura del puesto de trabajo docente, a aquellas condiciones vinculadas al género y al cuidado de los otrxs, las condiciones materiales de los hogares, la articulación con otras tareas extrapedagógicas asignadas por las instituciones relacionadas con la pandemia; como así también la diversidad de desafíos que ello supone en la heterogeneidad del territorio bonaerense.

Si bien aún no contamos con información suficiente respecto de lo que la heterogeneidad territorial significa en términos de desafíos para el trabajo pedagógico a distancia y la vuelta a clases; la expansión de la enfermedad en barrios populares brinda una anticipación de lo que el retorno a clases deberá considerar, así como la especificidad de los protocolos a los que habrá que atender frente a las condiciones sociosanitarias más críticas de barrios y de escuelas que aún no cuentan con agua de red o cloacas. Esto resulta central en este contexto de emergencia sanitaria donde el "cuidado" depende centralmente de dichas condiciones. También es insoslayable el modo como se ha evidenciado que las escuelas son parte de las políticas sociales de cuidado de infancias y adolescencias (centralmente a partir del SAE) y que la desigualdad en ese cuidado viola los derechos consagrados contra la discriminación de cualquier tipo frente a la educación.

De acuerdo con los datos producidos y relevados, es indudable que las mujeres desempeñaron como profesoras y como cuidadoras en sus hogares un lugar central, absorbiendo en gran medida la ausencia de políticas públicas de cuidado de menores en la pandemia, así como el acompañamiento a la escolarización en el hogar.

Esta "ceguera de género" en la política de continuidad pedagógica no tuvo correlato en otras dimensiones del cuidado, como la continuidad alimentaria que rápidamente se reestructuró para dar respuesta a la cuestión tal como ya se mencionó. 
En este sentido, es importante seguir observando las tensiones entre las condiciones sociosanitarias, la feminización de la docencia y de las tareas de cuidado de modo que permitan profundizar en una agenda abierta ya en nuestro país como lo es la distribución del cuidado y la responsabilidad pública y no individual frente al mismo. Aún queda mucho que recorrer y un próximo y complejo escenario de intermitencia en la vuelta a clases requerirá articular estas cuestiones para garantizar los derechos de todas y todos por parte del Estado.

\section{Bibliografía}

Agoff, S., Bertranou, J. y Foglia, C. (2016). Instituciones, política y burocracia en la formulación e implementación de políticas educativas en la provincia de Buenos Aires. En Bertranou, Isuani y Pereyra (editores.). ¿Unidad en la diversidad? Estudios sobre políticas públicas en la provincia de Buenos Aires. (pp. 271-312). Argentina: Universidad Nacional de General Sarmiento.

Arza, C. (2020). Familias, cuidado y desigualdad. En Cuidados y mujeres en tiempos de COVI-19. La experiencia en la Argentina. Documentos de Proyectos (LC/TS.2020/153), Santiago, Comisión Económica para América Latina y el Caribe (CEPAL).

Assusa, G. y Kessler, G. (2020). Reactivación de desigualdades y vulneración de derechos en tiempos de pandemia. En Bohoslavsky, J. P. (Ed.), Covid-19 y derechos humanos. La pandemia de la desigualdad. (pp. 93-107). Buenos Aires: Biblos.

Birgin, A. (1999). El trabajo de enseñar, Buenos Aires: Editorial Troquel.

Birgin, A. y Charovsky, M. (2013). Trayectoria de formación de futuros profesores en un territorio fragmentado. Pedagogía y Saberes, 39, 33-48, Bogotá: Facultad de Educación, Universidad Pedagógica Nacional.

CTERA (2020). Encuesta Nacional Salud y condiciones de trabajo docente en tiempos de emergencia sanitaria COVID19, Informe Final. Septiembre 2020. Recuperado de https://bit.ly/3fwdEYs

Dirección de Información y Estadística (2019). El Estado de la escuela. Datos e indicadores 2019. Provincia de Buenos Aires. Recuperado de https://bit.ly/3p0fYvm

Dirección Provincial de Estadística. (2020). Distribución del ingreso. Total seis aglomerados. Primer trimestre 2020. Ministerio de Hacienda y Finanzas. Recuperado de https://bit.ly/3utOFun

Gluz, N. y Rodríguez Moyano, I. (2015). Nuevas pretensiones en viejas institucionalidades: las condiciones de producción del trabajo docente en el contexto de las políticas de inclusión en la provincia de Buenos Aires. Ponencia presentada en el III Seminario Nacional de la Red ESTRADO Argentina, 2 al 4 de septiembre de 2015, Ensenada, Argentina.

Heargreaves A. (1998). Profesorado, Cultura y Posmodernidad. Madrid: Morata

INDEC (2010). Censo Nacional de Población, Hogares y Viviendas de 2010.Argentina: Ministerio de Economía y Finanzas Públicas.

INDEC (2020). Mercado de trabajo. Tasas e indicadores socioeconómicos. Ministerio de economía, Argentina.

Maceira, V., Ariovich, A., Crojetthovic, M, Jiménez, C. y Founier, M. (2020). El Conurbano en la cuarentena: Condiciones de vida y estrategias de los hogares. "Prevención y monitoreo del COVID-19 en municipios del Conurbano Bonaerense desde una perspectiva multidimensional". Proyecto de investigación apoyado por la Agencia I+D+i. Universidad Nacional General Sarmiento. Recuperado de https://bit.ly/3i47otR

Martinez, J. (1999) Trabajar en la escuela: profesorado y reformas en el umbral del siglo XX, Madrid: Miño y Davila. 
Martínez, D., Collazo, M. y Liss, M.I. (2009). Dimensiones del trabajo docente: una propuesta de abordaje del malestar y el sufrimiento psíquico de los docentes en la Argentina. Educação \& Sociedade, 30(107), 389-408.

Poliak, N. (junio, 2009). Percepciones de profesores de escuelas medias de la Ciudad de Buenos Aires en tiempos de fragmentación educativa. Trabajo presentado en el Congress of the Latin American Studies Association (LASA), Mesa: EDU 053, The Production of Subjectivitiy in the School. Río de Janeiro, Brasil

Provincia de Buenos Aires (2015). Ministerio de Economía. Encuesta Multipropósito Hogares, Ministerio de Economía.

Razavi, S. (2007). The political and social economy of care in a development context. Conceptual Issues, research questions and policy options, Gender and Development Programme. Paper 3. United Nations Research Institute for Social Development.

Rodríguez, C. (2010). La organización social del cuidado de niños y niñas en Argentina y Uruguay, Serie Mujer y Desarrollo, № 90, Santiago de Chile, CEPAL

Southwell, M. (2015). La escolarización en el Gran Buenos Aires. En G. Kessler (dir.). Historia de la Provincia de Buenos Aires: El Gran Buenos Aires. Buenos Aires: Unipe: Edhasa, Gonnet.

SUTEBA (2020). Encuesta Provincial de Trabajo Docente en contexto de Aislamiento Obligatorio. Primera presentación de resultado. Junio 2020. Disponible en https://bit.ly/3wEzpMO 